\title{
A VARIATIONAL PROBLEM WITH INEQUALITIES AS BOUNDARY CONDITIONS
}

\author{
BY JOHANNES C. C. NITSCHE ${ }^{1}$
}

\author{
Communicated by M. H. Protter, October 30, 1968
}

Variational problems, for the Dirichlet integral and other functionals, with inequalities as boundary conditions have been discussed by G. Fichera [3], J. L. Lions and G. Stampacchia [8], H. Lewy [6], H. Brezis et G. Stampacchia [1], H. Lewy and G. Stampacchia [7]. The question what happens when an object is pushed against a soap film leads to such a problem for the area integrand. While it can readily be demonstrated that a suitably chosen minimizing sequence tends to a limit surface, this surface merely is a weak solution of the problem which in reality possesses farther-reaching and characteristic regularity properties. It may be of interest to note (and can easily be verified by experiments with needles) that an obstruction consisting of one point, or even of a compact set of points of vanishing linear measure, is not capable of lifting the soap film. This follows from the general maximum principle in [2], [10]; see also [11], [12]. In H. Lewy's paper [6] the special case is considered where the obstructing object consists of a plane convex curve, and it is shown that the solution surface (minimizing Dirichlet's integral) actually is continuous everywhere and attaches itself to the obstruction along precisely one interval. The purpose of the present note is to report a similar result for the problem of minimizing the area integral over a convex domain which is symmetric with respect to the plane of the obstructing convex curve. Details of the proof, ${ }^{2}$ as well as applications of the method to more general cases, will appear elsewhere.

Let $P$ be a bounded (open) convex domain in the $(x, y)$-plane, symmetric with respect to the $x$-axis and on this axis containing the closed segment $\sigma_{0}=\{x, y ;|x| \leqq a, y=0\}$. Let $P_{0}$ be the set $P \backslash \sigma_{0}$ and $\bar{P}_{0}$ its closure; of course $\bar{P}_{0}=\bar{P}$. The boundary $\partial P$ of $P$ intersects the $x$-axis in the endpoints of an interval $\sigma=\left\{x, y ; c_{1} \leqq x \leqq c_{2}, y=0\right\}$, where $c_{1}<-a$ and $c_{2}>a$. Denote by $P^{+}$and $P^{-}$the intersections of $P$ with the half planes $y>0$ and $y<0$, respectively. On $\sigma_{0}$ a continuous

\footnotetext{
1 The preceding research was sponsored by the Air Force Office of Scientific Research under AFOSR Grant No. 883-67.

3 An account was given at the Mathematics Colloquium of the University of Minnesota on October 31, 1968 under the title, $A$ variational problem with inequalities as boundary conditions, or, How to fashion a cheap hat for Giacometti's brother.
} 
function $f(x)$ is given satisfying the conditions $f(-a)=f(a)=0$, $f(x) \not \equiv 0$ for $-a \leqq x \leqq a, f\left(x_{1}\right)+f\left(x_{2}\right) \leqq 2 f\left(\left(x_{1}+x_{2}\right) / 2\right)$ for $-a \leqq x_{1} \leqq x_{2} \leqq a$. Let $x_{0}$ be a point for which $f\left(x_{0}\right)=\max _{-a \leq x \leq a} f(x)$. We consider surfaces $U=\{z=u(x, y) ;(x, y) \in \bar{P}\}$, where the function $u(x, y)$ is continuous in $\bar{P}$, vanishes on $\partial P$, and satisfies the inequality $u(x, 0) \geqq f(x)$ on $\sigma_{0}$. The class of such surfaces will be called $\mathfrak{A}$.

TheOREM. The class $\mathfrak{A}$ contains a unique surface $\{z=u(x, y)$; $(x, y) \in \bar{P}\}$ of minimum area. For it the function $u(x, y)$ is continuous in $\bar{P}$, positive in $P$, real-analytic and a solution of the minimal surface equation in $P_{0}$. There is an interval $b_{1} \leqq x \leqq b_{2}$, where $-a<b_{1}<b_{2}<a$, such that $u(x, 0)=f(x)$ for $b_{1} \leqq x \leqq b_{2}$, while $u(x, 0)>f(x)$ for $-a \leqq x<b_{1}$ and $b_{2}<x \leqq a$.

The existence of a weak solution (which is not in class $\mathfrak{A}$ ) can be ascertained easily here. The difficulties in the proof of the continuity of this weak solution become surmountable if special discrimination is employed in the selection of the minimizing sequence. This is achieved by appropriate preliminary modifications. Each surface of a minimizing sequence is replaced by a polyhedral surface and this surface, in turn, by a more suitable one, either by cutting off " $(0, c)$ plus-regions" and " $(0, c)$-minus-regions" or by solving a discrete extremum problem. The latter surface is modified by an area reducing retraction process, e.g., the process of simultaneously cutting off " $(a, c)$-plus-regions" and " $(a, c)$-minus-regions." After these preparations a minimizing sequence $U_{n}=\left\{z=u_{n}(x, y) ;(x, y) \in \bar{P}\right\}, \quad(n$ $=1,2, \cdots)$, is obtained for which the curves $\mathbb{C}_{n}{ }^{(1)}=\left\{z=u_{n}(x, 0)\right.$; $\left.c_{1} \leqq x \leqq x_{0}\right\}$ and $\mathcal{C}_{n}{ }^{(2)}=\left\{z=u_{n}(x, 0) ; x_{0} \leqq x \leqq c_{2}\right\}$ are monotone and of bounded slope in each closed interval not containing the points $(-a, 0)$ and $(a, 0)$. Each function $u_{n_{k}}(x, y)$ of an appropriate subsequence is replaced in $P^{+}$and in $P^{-}$by the solution of the minimal surface equation with the same values on $\partial P^{+}$and $\partial P^{-}$. The passage to the limit can now be carried out. Using theorems from minimal surface theory, partly contained in [9], and an argument going back to A. Haar [4], it is shown that the limit surface has all the properties stated in the theorem.

The behavior of the solution surface near the points $\left(b_{1}, 0\right)$ and $\left(b_{2}, 0\right)$ of first contact with the obstruction depends on the regularity properties of the function $f(x)$. Some discussion of this matter can be found in [5].

\section{BIBLIOGRAPHY}

1. H. Brezis et G. Stampacchia, Sur la régularité de la solution d'inéquations elliptiques, Bull. Soc. Math France 96 (1968), 153-180. 
2. E. DeGiorgi and G. Stampacchia, Sulle singolarita eliminabili delle ipersuperficie minimali, Atti Accad. Naz. Lincei Rend. Cl. Sci. Fis. Mat. Natur. (8) 38 (1965), 352-357.

3. G. Fichera, Problemi elastostatici con vincoli unilaterali: il problema di Signorini con ambigue condizioni al contorno, Atti Accad. Naz. Lincei Mem. Cl. Sci. Fis. Mat. Natur. Sez. I (8) 7 (1964), 91-140.

4. A. Haar, Über reguläre Variationsprobleme, Acta Litt. Scient. Univ. Szeged 3 (1927), 224-234.

5. D. S. Kinderlehrer, Minimal surfaces whose boundaries contain spikes, Dissertation, University of California, Berkeley, 1968.

6. H. Lewy, On a variational problem with inequalities on the boundary, J. Math. Mech. 17 (1968), 861-884.

7. $\mathrm{H}$. Lewy and G. Stampacchia, On the regularity of the solution of a variational inequality, Comm. Pure Appl. Math. (to appear).

8. J. L. Lions and G. Stampacchia, Variational inequalities, Comm. Pure Appl. Math. 20 (1967), 493-519.

9. J. C. C. Nitsche, On new results in the theory of minimal surfaces, Bull. Amer. Math. Soc. 71 (1965), 195-270.

10. - Über ein verallgemeinertes Dirichletsches Problem für die Minimalflächengleichung und hebbare Unstetigkeiten ihrer Lösungen, Math. Ann. 158 (1965), 203-214.

11. - Zum allgemeinen Maximumprinzip, Math. Z. 92 (1966), 70-74.

12. The general maximum principle and removable singularities-a counterexample, Arch. Rational Mech. Anal. 22 (1966), 22-23.

University of Minnesota, Minneapolis, Minnesota 55455 